\title{
Co-registered spectral photoacoustic tomography and ultrasonography of breast cancer
}

Haixin Ke, Todd N. Erpelding, Alejandro Garcia-Uribe, Eileen Jacobs, Susan Holley, et al.

Haixin Ke, Todd N. Erpelding, Alejandro Garcia-Uribe, Eileen Jacobs, Susan Holley, Barbara Monsees, Lihong V. Wang, "Co-registered spectral photoacoustic tomography and ultrasonography of breast cancer," Proc. SPIE 8943, Photons Plus Ultrasound: Imaging and Sensing 2014, 89434G (3 March 2014); doi: $10.1117 / 12.2040591$

SPIE. Event: SPIE BiOS, 2014, San Francisco, California, United States 


\title{
Co-registered spectral photoacoustic tomography and ultrasonography of breast cancer
}

\author{
Haixin $\mathrm{Ke}^{1}$, Todd N. Erpelding ${ }^{2}$, Alejandro Garcia-Uribe ${ }^{1}$, Eileen Jacobs ${ }^{3}$, Susan Holley ${ }^{3}$, \\ Barbara Monsees ${ }^{3}$, and Lihong V. Wang ${ }^{1}$ \\ ${ }^{1}$ Department of Biomedical Engineering, Washington University in St. Louis, MO, USA \\ ${ }^{2}$ Philips Research North America, Briarcliff Manor, NY, USA \\ ${ }^{3}$ Washington University School of Medicine, St. Louis, MO, USA
}

\begin{abstract}
Many breast cancer patients receive neoadjuvant treatment to reduce tumor size and enable breast conserving therapy. Most imaging methods used to monitor response to neoadjuvant chemotherapy or hormone therapy depend on overall gross tumor morphology and size measurements, which may not be sensitive or specific, despite tumor response on a cellular level. A more sensitive and specific method of detecting response to therapy might allow earlier adjustments in treatment, and thus result in better outcomes while avoiding unnecessary morbidity. We developed an imaging system that combines spectral photoacoustic tomography and ultrasonography to predict breast neoadjuvant therapeutic response based on blood volume and blood oxygenation contrast. The system consists of a tunable dye laser pumped by a Nd:YAG laser, a commercial ultrasound imaging system (Philips iU22), and a multichannel data acquisition system which displays co-registered photoacoustic and ultrasound images in real time. Early studies demonstrate functional imaging capabilities, such as oxygen saturation and total concentration of hemoglobin, in addition to ultrasonography of tumor morphology. Further study is needed to determine if the co-registered photoacoustic tomography and ultrasonography system may provide an accurate tool to assess treatment efficacy by monitoring tumor response in vivo.
\end{abstract}

Keywords: Neoadjuvant chemotherapy, photoacoustic tomography, ultrasonography, breast cancer.

\section{INTRODUCTION}

Some breast cancer patients receive neoadjuvant treatment with chemotherapy or endocrine therapy before definitive surgery. The treatment aims to reduce tumor size in patients for whom breast conserving therapy would otherwise not be possible. Although it is hoped that neoadjuvant therapy would improve patient survival outcomes, data from clinical trials have not shown this benefit [1]. A sensitive method of detecting the primary tumor's response to a given therapy might allow earlier adjustment in treatment and result in better outcomes. Effective methods to monitor the early tumor response would also help limit unnecessary morbidity associated with patients not responding to therapy. Previous studies showed the feasibility of using optical tomography in monitoring tumor vascular changes and assessing tumor pathological response

Photons Plus Ultrasound: Imaging and Sensing 2014, edited by Alexander A. Oraevsky, Lihong V. Wang, Proc. of SPIE Vol. 8943, 89434G · C 2014 SPIE · CCC code: 1605-7422/14/\$18 · doi: 10.1117/12.2040591 
during neoadjuvant chemotherapy. Various predictors such as concentration (ct) of deoxyhemoglobin (ctHHb), oxy-hemoglobin, water $\left(\mathrm{ctH}_{2} \mathrm{O}\right)$, and bulk lipid, were assessed [2]-[4].

Photoacoustic Tomography (PAT) is a non-ionizing and noninvasive hybrid imaging technique that can measure strong optical absorption contrasts with high ultrasonic spatial resolution at depths beyond the optical diffusion limit [5] [6]. Both image resolution and depth are highly scalable with the ultrasonic frequency. Photoacoustic imaging relies on the photoacoustic effect to generate pressure waves that can be detected by conventional ultrasound array transducers. Therefore, it is highly compatible with ultrasound imaging. The same detection mechanism greatly simplifies spatial registration between photoacoustic and ultrasound images. Photoacoustic imaging is an emerging modality that could expand the scope of diagnostic ultrasonography into new clinical applications, such as image-guided sentinel lymph node biopsy, breast cancer diagnosis, and therapy monitoring.

We developed a dual-modality system that combines PAT and ultrasonography. The system can provide co-registered PAT and ultrasound images that reveal both morphological and functional information. Therefore, the technology described represents a potential new way to evaluate response to therapy, and may be capable of detecting response earlier than conventional imaging techniques, such as mammography and ultrasound (US), that can detect a change in tumor morphology.

With the help of ultrasonography for identifying the tumor region, we demonstrated the capability of the PA technique to monitor in vivo blood oxygen saturation in breast tumors. Repeated imaging of the same patient was performed to evaluate the possibility of monitoring the treatment progress. Further study is needed to determine if the combination of the functional as well as morphological information obtained by both modalities can provide more accurate evaluation of the treatment progress and efficiency.

\section{MATERIALS AND METHODS}

\subsection{Imaging system}

The dual-modality imaging system integrates a laser system into a clinical US imaging scanner. A system diagram is shown in Fig. 1(a). The commercial US scanner was modified to allow access to raw per-channel RF acoustic data, while all imaging capabilities of the commercial US scanner were retained [7]. The laser system consists of a tunable dye laser (PrecisionScan-P, Sirah) pumped by a Q-switched Nd:YAG laser (PRO-350-10, Newport). We designed a photoacoustic transducer that integrates the fiber output into a commercial ultrasound linear array transducer (L8-4, Phillips), as shown in Fig. 1(b). The optical fiber bundles flank both sides of the ultrasound array transducer. Photoacoustic waves are received by the ultrasound transducer in the same geometry as pulse-echo ultrasound. The custom-made data acquisition system controls the triggering of both modalities and collects raw data for image display and post-processing. PA images are reconstructed using a delay-and-sum beam-forming algorithm implemented in FPGA firmware, generating cross-sectional PA B-mode images that can be fused into corresponding US images displayed in real-time at 5 frames per second [8].

The oxygen saturation was calculated using the method described in [9]. To calculate the oxygen saturation, we need to acquire PA images at multiple wavelengths. The system can 
operate in two different modes to acquire and display spectroscopic PA/US images. First, the system can tune the dye laser to multiple, user-defined preset wavelengths while PA images are acquired. Second, we modified the pump Nd:YAG laser to get access to the fundamental laser $(1064 \mathrm{~nm})$. A mechanical shutter is synchronized to the laser trigger and alternatively blocks the dye output or the fundamental output on consecutive laser shots. Therefore, the PA imaging can also operate in a fast-switching, dual-wavelength mode with one wavelength from the dye laser and the other wavelength at $1064 \mathrm{~nm}$. Based on the spectra of oxy- and deoxy-hemoglobin [10], we chose $782 \mathrm{~nm}$ from the dye laser and $1064 \mathrm{~nm}$ from the Nd:YAG laser for the dualwavelength mode. The two wavelengths are routed to the same path by a dichroic mirror before they enter the fiber bundle. A beam sampler is placed just before the fiber so that a small amount of the laser is reflected to a photodiode to measure the pulse-to-pulse laser power fluctuations for subsequent compensation.

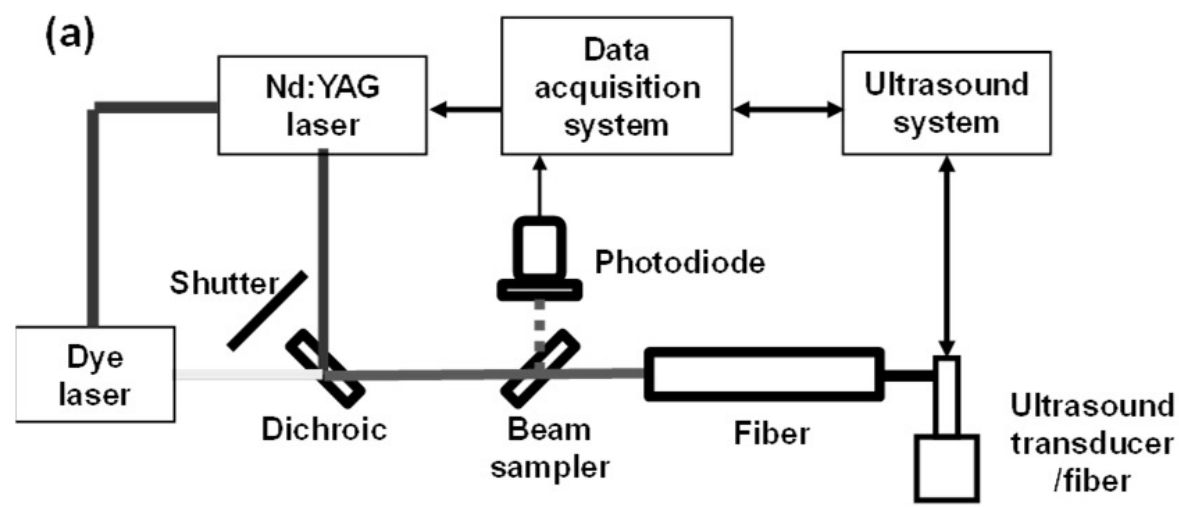

(b)

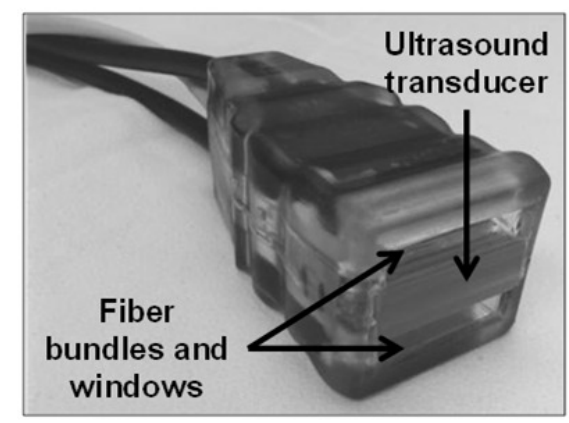

Fig.1. (a) Schematics of the dual-modality imaging system. (b) A photograph of the integrated transducer.

\subsection{Clinical study}

Patients enrolled in this study must be newly diagnosed with clinical stage II or III biopsyproven breast cancer (initial diagnosis), or have relapsed/recurrent stage II or III biopsy-proven breast cancer, and be considered eligible for breast surgery. They must be preparing to undergo neoadjuvant therapy (chemotherapy or endocrine/hormone therapy) prior to definitive surgical 
interventions. Each participant will undergo imaging at three separate times: 1) prior to initiation of neoadjuvant therapy; 2) during neoadjuvant therapy, probably at two weeks after initiation of therapy; and 3) at a later point in time to be determined from the results of Phase I (and possibly Phase II), but prior to definitive surgery.

During each visit, the total imaging procedure included three steps. First, standard ultrasound measurements of the tumor were made by the operator to locate the tumor and record the tumor dimensions. Second, we scanned across the tumor region with interleaving US and PA imaging in both longitudinal and transverse directions. This scanning measurement gave us a full view of the tumor area, and at the same time allowed us to identify critical features on the co-registered US and PA images. These features were not only the targets for oxygen saturation measurements, but also for monitoring longitudinal changes. Third, after we found good targets, we did spectral PA measurements over these targets, during which the transducer position was fixed.

\section{RESULTS AND DISCUSSION}

Each patient was imaged before her neoadjuvant treatment started, and then during her treatment (17 days after the first visit), following the same imaging procedure. Given the stronger laser power and lesser melanin absorption from the skin at $1064 \mathrm{~nm}$, the PA images at $1064 \mathrm{~nm}$ were used to find critical features and select the targets for oxygen saturation measurements.

The imaging results of one patient's first visit are shown in Fig. 2. During the scanning measurement, PA and US data were collected alternatively. At the same time, they were processed to overlay PA and US images so that we could better interpret the images. The PA, US and fused images could be shown in real time at 5 frames/s. Fig. 2(a) shows one frame during the scan in the longitudinal direction. The tumor boundary was identified by US imaging. A vessel-like structure was clearly shown in the US image, which also generated a strong PA signal on the corresponding PA image, suggesting a large blood vessel lying near the tumor. The transducer was then fixed at roughly the same position at which this frame was collected, and the PA spectral measurement was performed. Fig. 2(b) shows a PA image when the laser wavelength was $1064 \mathrm{~nm}$. Comparing this image to the fused PA/US image helped identify a couple of other blood spots on the boundary of and inside the tumor. We chose three regions to calculate the oxygen saturation, with the data collected in dual-wavelength mode. PA signals in these regions were thresholded and spatially averaged over neighboring pixels.

The imaging results of the patient's second visit are shown in Fig. 3. The same blood vessel and similar tumor shape as in Fig. 2 were found in the PA/US image in Fig. 3(a). Similar features on the boundary of and inside the tumor can be found on the thresholded PA image in Fig. 3(b), although the positions were slightly changed. The same three regions were chosen to calculate the oxygen saturation, and they had similar values to those from the first visit.

\section{CONCLUSIONS}

We developed a dual-modality imaging system that integrates PAT and ultrasonography, and applied it to study patients undergoing neoadjuvant therapy for breast cancer. We demonstrated 
that the system provides the functional imaging capabilities provided by PAT, such as oxygen saturation, in addition to ultrasonography of tumor morphology. Our initial results also showed the importance of combining functional PA and morphological US images to identify critical features in tumors. Further study is needed to determine if co-registered photoacoustic tomography and ultrasonography system can provide an accurate tool to assess treatment efficacy by monitoring tumor response in vivo.
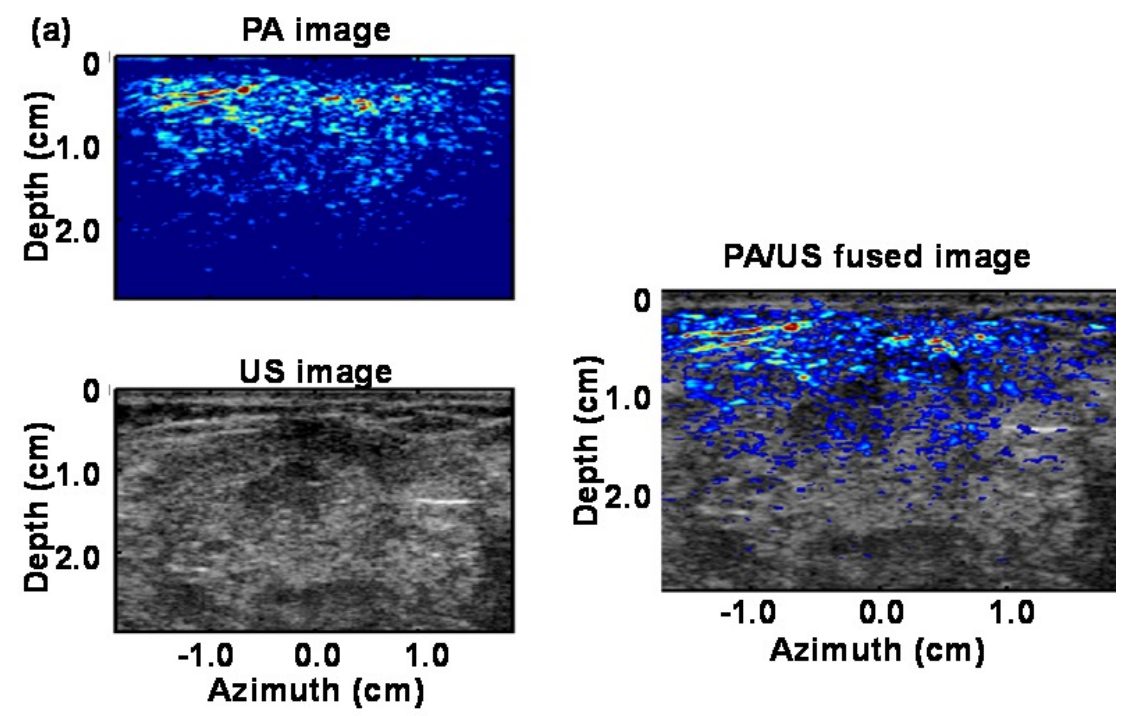

(b)

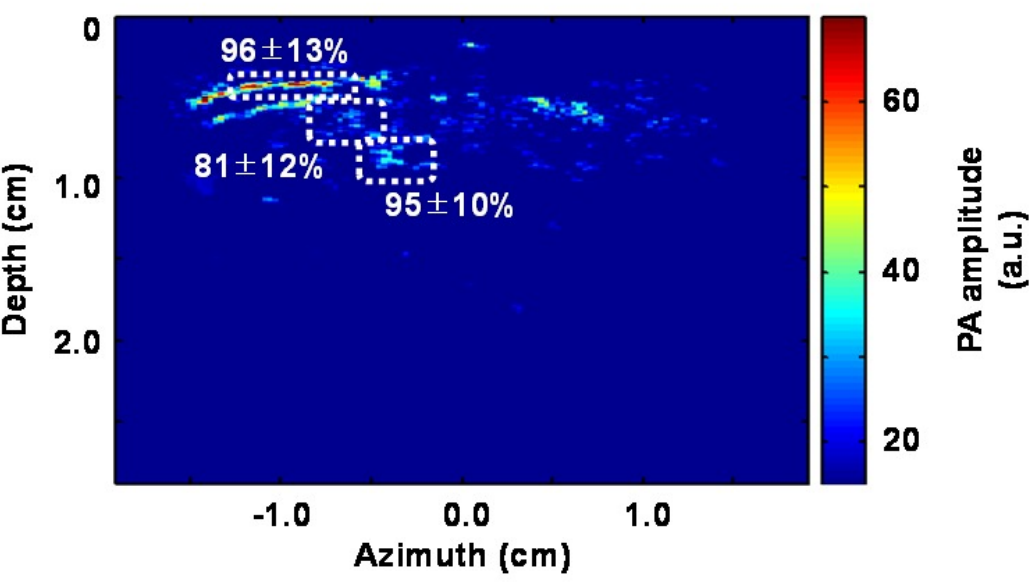

Fig.2. The imaging results of the first visit. (a) The PA and US images acquired during the scan in the longitudinal direction, as well as the overlay of the two images. (b) The threshold PA image at $1064 \mathrm{~nm}$. The dashed the boxes show the three regions over which the averaged oxygen saturation were calculated. The values (mean \pm standard derivation) are shown in the figure. 

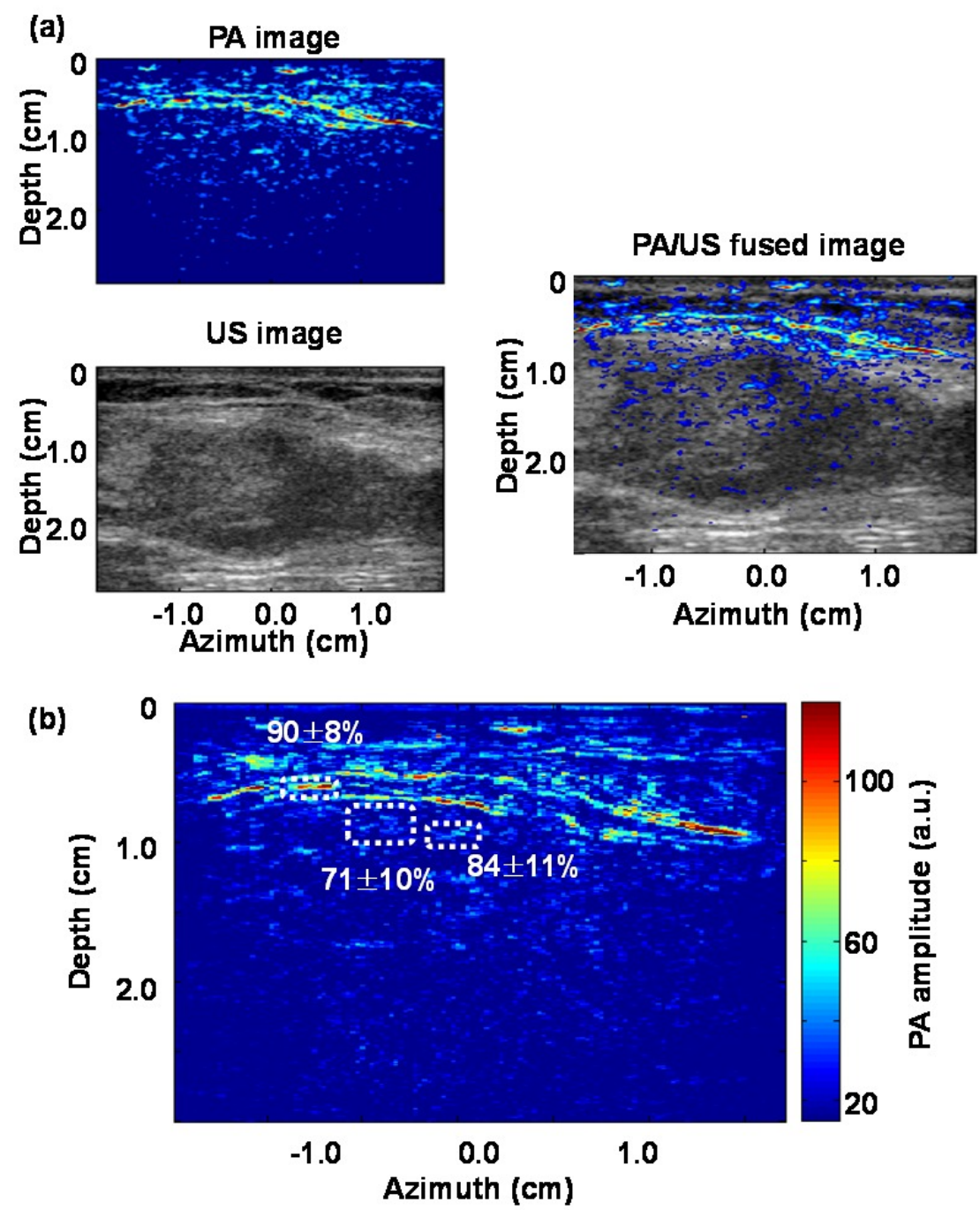

Fig.3. The imaging results of the second visit. (a) The PA and US images acquired during the scan in the longitudinal direction, as well as the overlay of the two images. (b) The threshold PA image at $1064 \mathrm{~nm}$. The dashed the boxes show the three regions over which the averaged oxygen saturation were calculated. The values (mean \pm standard derivation) are shown in the figure.

\section{ACKNOWLEDGEMENTS}

This work was sponsored by NIH grants R01 CA134539, DP1 EB016986 (NIH Director's Pioneer Award), R01 EB016963, and U54 CA136398. L. V. Wang has a financial interest in Endra, Inc., and Microphotoacoustics, Inc., which, however, did not support this work. 


\section{REFERENCES}

[1] D. Mauri, N. Pavlidis and J. P. A. Ioannidis, "Neoadjuvant Versus Aduvant Systemic Treatment in Breast Cancer: A Meta-Analysis,” Journal of the National Cancer Institute 97(3), 188-94 (2005).

[2] Q. Zhu, S. Tannenbaum, P. Hegde, M. Kane, C. Xu, and S. H. Kurtzman, "Noninvasive mornitoring of breast cancer during neoadjuvant chemotherapy using optical tomography with ultrasound localization," Neoplasia 10(10), 1028-1040 (2008).

[3] A. Cerussi, D. Hsiang, N. Shah, R. Mehta, A. Durkin, J. Butler, and B. J. Tromberg, "Predicting response to breast cancer neoadjuvant chemotherapy using diffuse optical spectroscopy," Proceedings of the National Academy of Sciences 104(10), 4014-4019 (2007).

[4] B. J. Tromberg, A. Cerussi, N. Shah, M. Compton, A. Durkin, D. Hsiang, J. Butler, and R. Mehta, "Diffuse optics in breast cancer: detecting tumors in pre-menopausal women and monitoring neoadjuvant chemotherapy,” Breast Cancer Research 7(6), 279-285 (2005).

[5] L. V. Wang, "Prospects of photoacoustic tomography," Medical Physics 35(12), 5758-5767 (2008).

[6] M. Xu and L. V. Wang, "Photoacoustic imaging in biomedicine," Review of Scientific Instruments 77, 041101 (2006).

[7] T. N. Erpelding, C. Kim, M. Pramanik, L. Jankovic, K. Maslov, Z. Guo, J. A. Margenthaler, M. D. Pashley, and L. V. Wang, "Sentinel Lymph Nodes in the Rat: Noninvasive Photoacoustic and US Imaging with a Clinical US System,” Radiology 256(1), 102-110 (2010).

[8] K. P. Köstli, M. Frenz, H. Bebie, H. P. Weber, "Temporal backward projection of optoacoustic pressure transients using fourier transform methods," Physics in Medicine and Biology 46(7), 1863-1872 (2001).

[9] M. Sivaramakrishnan, K. Maslov, H. F. Zhang, G. Stoica, and L. V. Wang, "Limitations of quantitative photoacoustic measurements of blood oxygenation in small vessels," Physics in Medicine and Biology 52(5), 1349-1361 (2007).

[10] W. G. Zijlstra, A. Buursma, and O. W. van Assendelft, "Visible and near infrared absorption spectra of human and animal haemoglobin determination and application", CRC press, (2000). 